\title{
Head and neck cancer in primary care: presenting symptoms and the effect of delayed diagnosis of cancer cases
}

\author{
Olli-Pekka Alho, Heikki Teppo, Pekka Mäntyselkä, Saara Kantola
}

\section{ABSTRACT}

Background: Little is known about the diagnosis of head and neck carcinoma in primary care. We sought to estimate the general prevalence of symptoms reported by patients with head and neck carcinomas and to determine the association between detection patterns of head and neck cancer cases in primary care and survival.

Methods: In a cross-sectional survey, we used a questionnaire to estimate the general prevalence of symptoms associated with head and neck cancer from a sample of 5646 primary care visits in 25 randomly selected health centres over 4 weeks throughout Finland. A population-based retrospective cohort study involved the 221 patients resident in one primary health care district (population about 700000 ) in whom head and neck carcinoma was diagnosed between Jan. 1, 1986, and Dec. 31,1996 . Data on the initial primary care visit, clinical characteristics and survival were obtained from patient charts.

Results: Of 5646 visits to a primary care practitioner, $11 \%$ (617) were made because of the same symptoms as those initially reported by patients later found to have head and neck cancer. According to the cohort data, the detection rate of these carcinomas in primary care was 1 per $\sigma_{3} 000$ visits. At the initial visit of 221 patients later found to have cancer, $56 \%$ (123) received referrals, $24 \%$ (53) follow-up appointments and $20 \%$ (45) neither ("overlooked"). At 3 years, the risk of death was significantly higher among patients whose disease was overlooked (adjusted hazard ratio [HR] 1.89, $95 \%$ confidence interval $[\mathrm{Cl}] 1.03-3.45)$. The excess risk associated with being overlooked, however, was confined to subjects with tongue or glottic tumours ( $\mathrm{HR} 4.25,95 \% \mathrm{Cl}$ 1.59-11.4) (number needed to harm 3.0, 95\% Cl 1.9-6.7).

Interpretation: Despite the rarity of patients with head and neck carcinoma in primary care, patients with symptoms of these diseases and especially with symptoms of tongue and glottic carcinomas should be initially referred for further care or followed up.

$C M A J 2006 ; 174(6): 779-84$

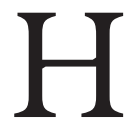
ead and neck cancer usually manifests as squamous cell carcinomas of the upper aerodigestive tract. ${ }^{1}$ The rates of death associated with head and neck cancer continue to be high (a 5-year relative survival rate of $33 \%-62 \%$ according to the site of the tumour) be- cause the disease is often undiagnosed until it is at an advanced stage. ${ }^{2-4}$ Whether and to what extent this is due to failures in the initial diagnosis in primary care is unknown.

The research on head and neck cancer has generally lacked a primary care perspective. Statistics describing the overall occurrence of cases (e.g., they constitute about $5 \%$ of all cancers in the West; some 40 ooo new cases occur each year in the United States) $)^{1,5}$ fail to give a good idea of how often a general practitioner sees a new case. Similarly, the main symptoms are well known, ${ }^{1,6}$ but how often they occur is not. Misdiagnoses are frequent, ${ }^{7-9}$ but it is unclear whether this is because physical examination of the region is technically demanding, particularly the laborious indirect visualization of the nasopharynx and larynx with small mirrors. ${ }^{10}$ Finally, it is also unclear whether an initial misdiagnosis leads to poorer outcomes. Attempts to link long delays in diagnosis with advanced stages of the disease or poor survival have had conflicting results. ${ }^{8,11-14}$

We sought to investigate the diagnosis of head and neck carcinoma in primary care by performing 2 studies. In a crosssectional questionnaire study, we explored the frequency of symptoms associated with head and neck carcinoma. In a population-based cohort of 22I patients, we sought answers to the following questions: How often does a general practitioner see a new case? How are the patients examined? And, finally, does the outcome of the initial visit affect survival?

\section{Methods}

In the questionnaire study, we used cross-sectional data ${ }^{15}$ to gather information on the frequency of the symptoms of head and neck cancer in primary care in Finland. A total of 25 health centres were randomly selected throughout Finland ( 4 in cities, 5 in smaller towns and I6 in rural communities). Altogether, 28 primary care physicians from these centres took part in a 4week study, for I week in each of the 4 seasons in I996. Age, sex and the primary presenting symptom ${ }^{16}$ were recorded for all patient visits except visits received out of regular business hours.

In the cohort study, we used a population-based retrospective cohort design to collect data on the cancer patients. The area where the cancer patients were identified comprises 87 communities (total population about 700 ooo), which maintain one primary health care centre each and 4 central hospitals and one university hospital collectively. According to the Finnish Medical Association, there were 520 physicians and I 502800 visits for primary care services in the study area in 1996 . All of the patients in whom tongue, pharyngeal or laryngeal cancer 
was diagnosed (International Classification of Diseases, ninth and tenth revisions, codes I4I, I46-I48, I6I, Co2, Co9-CII, $\mathrm{C}_{13}, \mathrm{C}_{32}$ ) between Jan. I, I986, and Dec. 3I, 1996, were identified from the registers of Oulu University Hospital, where all such cases were assumed to be treated. Only cases of histologically verified squamous cell carcinoma were included. We found 318 new cases, and to confirm that our sample was populationbased, we cross-checked our cases with those of the national Finnish Cancer Registry, whose files are practically complete. ${ }^{17}$ Our cases accounted for $90 \%$ of the 353 cases registered in the Finnish Cancer Registry in the respective area and period.

Each patient's medical and dental charts in the primary health care centre and local private practices were searched for data on the initial visit that subsequently resulted in the diagnosis of cancer. Data on the following variables were collected: primary reason for presentation, duration of symptoms (days), specialization of primary care practitioner (physician; dentist; ear, nose and throat specialist), physical examinations done (inspection of mouth, indirect laryngoscopy, posterior rhinoscopy, palpation of neck), diagnosis made (suspicion of cancer, benign tumour, infection, no diagnosis) and outcome of the visit (referral, scheduled follow-up visit, neither). For the purposes of this study, we considered patients who received neither a referral nor a scheduled follow-up visit to have been "overlooked." From the medical charts of the university hospital, we collected information on the primary site (tongue, pharynx, larynx) and subsite (tongue, oro-, naso- and hypopharynx, glottis, supraglottis) of the tumour ${ }^{1}$ and cancer stage (I-IV). ${ }^{18}$ From either primary care or hospital charts, we collected data on the patient's current or former tobacco use and socioeconomic status ${ }^{19}$ (high: employers, self-employed people, higher-level employees; low: lower-level employees, manual workers, and others, including students and people receiving pensions). Dates and causes of death were obtained from Statistics Finland. The Finnish Ministry of Social Affairs and Health granted permission to collect these data.

The treatment of head and neck cancer cases at the university hospital is planned in a joint clinic meeting with radiation oncologists and head and neck and plastic surgeons. Treatment is based on the clinical stage and location of the tumour and follows suggested guidelines. ${ }^{1}$ Options for treatment of the primary tumour include conventional or laser surgery and radiotherapy. In more advanced cases, the tumour area is irradiated postoperatively, followed by tumour dissection or neck irradiation.

The health care system in Finland is based on a general health insurance scheme and provides equal access to medical services for all citizens. Municipalities are responsible for health care, which is paid for by tax revenues. All patients must first present in the primary care service, and no one can be admitted to hospital without a referral letter from a physician working in primary care. Finnish law obliges all licensed physicians to keep medical records of each medical visit. Physicians record each visit on a sheet with the following subheadings: reason for the visit, medical history, physical examination, diagnosis, treatment and possible referral. Medical training in Finland includes theoretical and practical instruction in how to make a thorough ear, nose and throat examination using a head lamp.
We reported values as means and standard deviations or medians and ranges for descriptive purposes. The $\chi^{2}$ and MannWhitney $U$ tests were performed to analyze associations between 2 variables. We constructed survival curves as they related to the outcome of the initial visit according to the Kaplan-Meier method, starting from the date of the histologic diagnosis. ${ }^{20} \mathrm{We}$ did not classify patients according to the length of the delay to diagnosis to avoid bias due to unequal tumour growth rate. ${ }^{21,22}$ Slowly growing tumours with a good prognosis may have long delays to diagnosis and rapidly growing tumours with a poor prognosis short delays regardless of how they are detected. Although the tumour growth rate may have been dissimilar in different diseases, here the tumour growth rate may be regarded as an intermediate factor, not a confounding one. The adverse impact of delayed diagnosis on survival may be attributable to a lead time effect if survival is measured from the time of diagnosis. ${ }^{22}$ In other words, for patients whose case has a poor prognosis, the interval between the start of treatment and death will be shorter if treatment is started later in the course of the disease. To control for this, we also calculated survival from symptom onset. The primary end point was tumour-related death, but the unadjusted hazard ratio was calculated using all-cause death as the end point as well.

Differences between the groups were tested with the logrank test. ${ }^{23}$ We calculated the absolute difference and the $95 \%$ confidence intervals (CIs) in proportions of death between the groups and a respective time-specific number needed to harm at 3 years. ${ }^{24} \mathrm{~A}$ Cox proportional-hazards regression model was used for the multivariate analysis. ${ }^{25}$ The proportional hazards assumption was assessed by a plot of $\log (-\log$ (survival function)) versus time for the primary end point. The cumulative risk of dying of a tumour-related cause was calculated as a hazard ratio with 95\% CIs, which was adjusted for age (years), sex, socioeconomic status (low v. high), duration of symptoms (days), cancer stage (I-IV) and study period (per year).

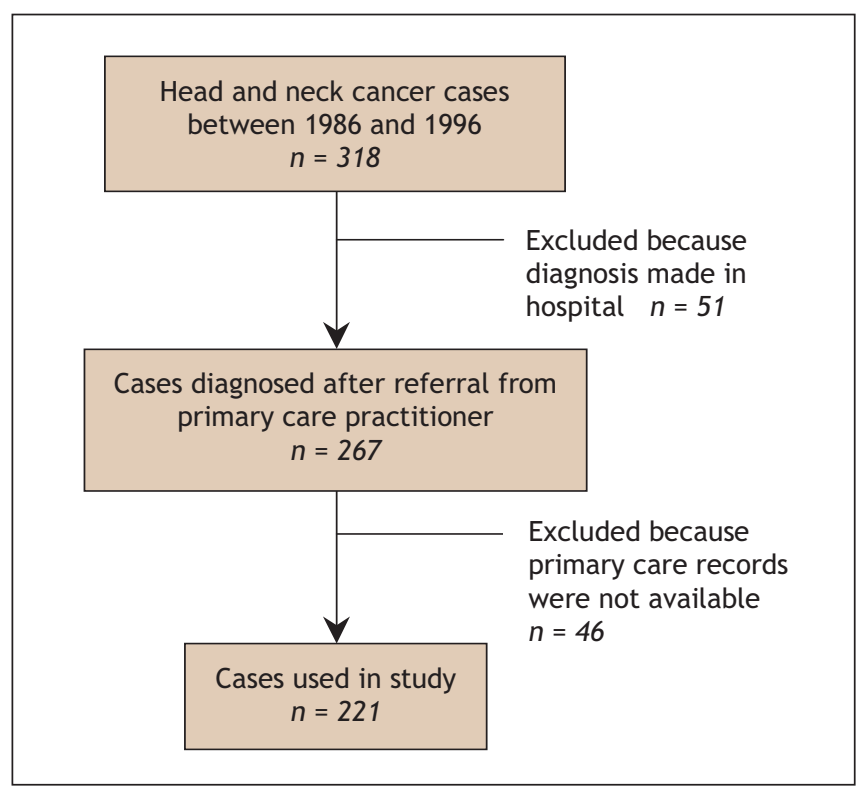

Fig. 1: Flow of participants through the cohort study. 
Multivariate modeling was done separately for patients with tongue and glottic tumours, since these lesions tend to cause symptoms earlier than other head and neck tumours. ${ }^{1}$

\section{Results}

The questionnaire data included 5646 primary care visits, of which $62 \%(3500)$ were made by women. In the cohort study, we found 318 patients with head and neck cancer between 1986 and 1996 , and after 97 patients were excluded, a cohort of 22I patients was left (of whom 62 had tongue carcinoma, 66 pharyngeal carcinoma and 93 laryngeal carcinoma) (Fig. I).

The primary tumour symptoms reported by the cancer patients at their initial primary care visit (cohort data) and the number of patients in the general population reporting the same symptoms (questionnaire data) are shown in Table I. Of the I22 patients with tongue or glottic carcinoma, 94 (77\%) presented with a prolonged pain or change in the tongue or persistent hoarseness, whereas patients with tumours at other sites had more variable symptoms (data not shown). According to the questionnaire data, symptoms similar to those of cancer patients were reported by patients in the general population at 617 (II\%) of the 5646 primary care visits (Table I).

The mean age of the carcinoma patients (cohort data) was 63 (standard deviation $\mathrm{I} 2$ ) years, 156 (71\%) were men, and $\mathrm{I} 6 \mathrm{I}$ $(73 \%)$ were current or former users of tobacco (Table 2). The mean duration of follow-up after diagnosis was 5.9 (range 0-I7.9) years. On average, 24 (267/II) new cases emerged per year during this study, giving a detection rate of 24/I 502800 (i.e., one per some 63 ooo visits in primary care, or an average of one case in 22 years for one physician).
Of the 22I carcinoma patients, I23 (56\%) were referred for further care after their initial visit, 53 (24\%) were scheduled for follow-up, and no action followed the visit of $45(20 \%)$. The physician delay (time from the initial presentation to diagnosis) was significantly longer in the cases of patients whose disease was overlooked compared with the referred or followed-up patients (median [range] I37 [20-2256] v. 3I [2-706] days, $p<$ 0.001 ). The only background factors associated with the disease being overlooked were the patient's low socioeconomic status, short duration of symptoms (patient delay) and insufficient physical examination (Table 2). Patients whose disease was overlooked had a significantly shorter time from diagnosis to disease-specific death than patients who were referred or followed up (Fig. 2) (unadjusted hazard ratio [HR] at 3 years I.80 [95\% CI I.08-2.99]). However, the excess risk of death associated with being overlooked was present only in patients with tongue or glottic carcinoma (Table 3) (unadjusted HR 4.60, $95 \% \mathrm{CI}$ 2.03-I0.4); the number needed to harm by overlooking the disease was 3.0 (95\% CI I.9-6.7). These patients tended to present early in the course of the disease. The effect remained significant after adjustment for other prognostic factors (Table 3). Both the calculation of tumour-specific survival from symptom onset and the use of all-cause death as the end point diminished the effect only slightly (unadjusted HRs 3.28 , 95\% CI I.53-7.03 and 2.56, 95\% CI I.24-5.29, respectively).

\section{Interpretation}

These results show concretely both how rare head and neck carcinoma is in primary care and how common are its presenting symptoms. We calculated that, in Finland, primary

Table 1: Primary symptom reported at initial primary care visit in a cohort of patients with head and neck carcinoma and by patients in the general population

\begin{tabular}{|c|c|c|c|c|}
\hline \multirow[b]{2}{*}{ Primary symptom* } & \multirow{2}{*}{$\begin{array}{c}\text { Patients with } \\
\text { head and neck } \\
\text { carcinoma, no. (\%) } \\
n=221\end{array}$} & \multicolumn{3}{|c|}{$\begin{array}{l}\text { Patients and their characteristics reporting } \\
\text { the same symptoms in primary care visits } \dagger \\
\qquad n=5646\end{array}$} \\
\hline & & No. (\%) & Mean age (SD), yr & Men, no. (\%) \\
\hline Hoarseness & $61(28)$ & $24(0.4)$ & $35(20)$ & $8(33)$ \\
\hline Throat pain & $45(20)$ & $150(2.7)$ & $32(20)$ & $52(35)$ \\
\hline Change in the tongue & $24(11)$ & $4(<0.1)$ & 13 (19) & $1(25)$ \\
\hline Pain in the tongue & $21(10)$ & $20(0.3)$ & $52(21)$ & $5(25)$ \\
\hline Neck lump & $14(6)$ & $8(0.1)$ & $23(24)$ & $4(50)$ \\
\hline Dysphagia & $6 \quad(3)$ & $5(<0.1)$ & $52(12)$ & $2(40)$ \\
\hline Dyspnea & $5 \quad(2)$ & $69(1.2)$ & $55(25)$ & $29(42)$ \\
\hline Tussis or hemoptysis & $4 \quad(2)$ & $275(4.9)$ & $34(26)$ & $114(41)$ \\
\hline General symptoms & $2(1)$ & $62(1.1)$ & $56(23)$ & $25(40)$ \\
\hline Other local symptoms & $7 \quad(3)$ & - & - & - \\
\hline Unrelated symptoms & $32(15)$ & - & - & - \\
\hline Total & $221(100)$ & $617(11)$ & - & - \\
\hline
\end{tabular}

Note: SD = standard deviation

*These symptoms were classified according to ref. 18 in the following categories (from top to bottom): R23 (voice symptoms); R21 (throat symptoms); D83 (disease of mouth/tongue/lip); D20 (mouth/tongue/lip symptoms); B02 (enlarged lymph nodes); D21 (difficulty in swallowing); R02 (dyspnea); R05 (cough); A04, A05, T08 (weakness/fatigue/decline in overall condition/weight loss). †In 25 randomly selected primary health care centres. 
care physicians would encounter an average of 2 new cases during their entire career, but that some $\mathrm{II} \%$ of all visits in primary care were made for the same symptoms experienced by carcinoma patients. Still, the correct diagnosis in primary care was important, since we observed a significant increase in the risk of tumour-related death associated with the disease being overlooked at initial presentation. The excess risk, however, was confined only to subjects with tongue or glottic tumours. We calculated that overlooking the disease was associated with one extra death for every 3 such patients. Ac- cording to our findings, it was the initial decision not to refer or follow-up that was related to poor survival, but that the initial misdiagnosis was not harmful if scheduled follow-up was arranged.

The presenting symptoms and distribution of different head and neck tumours reported in our study are in line with those of earlier reports. ${ }^{1,26}$ Our finding that initial lack of referral or follow-up was strongly associated with impaired survival in tongue and glottic carcinomas but not in pharyngeal and supraglottic carcinomas is in agreement with the find-

Table 2: Characteristics of patients with head and neck carcinoma by outcome of the initial visit in primary care

\begin{tabular}{|c|c|c|c|}
\hline \multirow[b]{2}{*}{ Characteristic } & \multicolumn{2}{|c|}{ Patients by outcome, no. (\%)* } & \multirow[b]{2}{*}{$p$ value $\uparrow$} \\
\hline & $\begin{array}{c}\text { Referred or } \\
\text { followed up } \\
\quad n=176\end{array}$ & $\begin{array}{c}\text { Overlooked } \\
\quad n=45\end{array}$ & \\
\hline Age, mean (SD), yr & $64(12)$ & $61(13)$ & 0.14 \\
\hline Sex, male & $128(73)$ & $28(62)$ & 0.17 \\
\hline Low socioeconomic status $\ddagger^{21}$ & $121(72)$ & $36(88)$ & 0.04 \\
\hline Living independently at home $\neq$ & $166(95)$ & $43(96)$ & 0.88 \\
\hline Current or former tobacco use $\neq$ & $130(76)$ & $31(74)$ & 0.76 \\
\hline Tumour-related reason for presentation & $150(85)$ & $39(87)$ & 0.81 \\
\hline Median patient delay (range), $\mathrm{d}$ & $60(0-1827)$ & $30(0-700)$ & 0.008 \\
\hline Median physician delay (range), $\mathrm{d}$ & $31(2-706)$ & $137(20-2256)$ & $<0.001$ \\
\hline \multicolumn{4}{|l|}{ Physical examination } \\
\hline Inspection of mouth & $157(89)$ & $37(82)$ & 0.20 \\
\hline Indirect laryngoscopy & $80(45)$ & $8(18)$ & 0.001 \\
\hline Palpation of neck & $33(19)$ & $6(13)$ & 0.39 \\
\hline Posterior rhinoscopy & $17(10)$ & $5(11)$ & 0.77 \\
\hline Site of tumour examined & $145(82)$ & $23(51)$ & $<0.001$ \\
\hline Primary site of tumour & & & 0.17 \\
\hline Tongue & $49(28)$ & $13(29)$ & \\
\hline Pharynx & $48(27)$ & $18(40)$ & \\
\hline Larynx & $79(45)$ & $14(31)$ & \\
\hline Cancer stage at diagnosis ${ }^{20}$ & & & 0.36 \\
\hline I & $42(23)$ & $6(11)$ & \\
\hline II & $36(21)$ & $11(25)$ & \\
\hline III & $52(30)$ & $14(32)$ & \\
\hline IV & $46(26)$ & $14(32)$ & \\
\hline Diagnosis made in primary care & & & $<0.001$ \\
\hline Suspicion of cancer & $95(54)$ & $0(0)$ & \\
\hline Benign disease & $17(10)$ & $6(13)$ & \\
\hline Infection & $41(23)$ & $30(67)$ & \\
\hline None & $22(13)$ & $9(20)$ & \\
\hline Primary care practitioner & & & 0.41 \\
\hline Physician & $152(86)$ & $42(93)$ & \\
\hline Dentist & $12(7)$ & $2(4)$ & \\
\hline Ear, nose and throat specialist & $12(7)$ & $1(2)$ & \\
\hline
\end{tabular}

*Unless stated otherwise.

$\dagger \chi^{2}$ test in categorized variables and Mann-Whitney $U$ test in continuous variables.

$\neq$ Percentages calculated among those data available. 
ings that long physician delay is related to decreased survival in laryngeal carcinoma ${ }^{14}$ but not in pharyngeal ${ }^{12}$ or oropharyngeal carcinoma. ${ }^{11}$ There is no difficulty in getting a return appointment with a physician in our health care system, and thus this does not explain the long physician delay and poor outcomes found here for patients whose disease was overlooked. A lack of diagnosis at the initial visit may give patients a false sense of security that leads them to postpone the second consultation. Tromp and associates found that a lack of

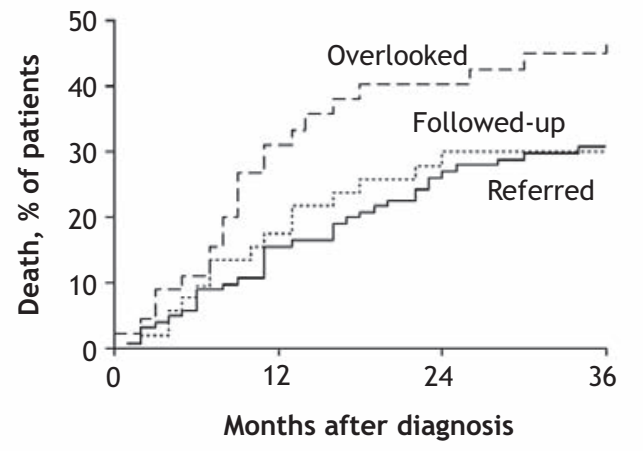

No. at risk of death

$\begin{array}{lrrrr}\text { Referred } & 123 & 100 & 80 & 71 \\ \text { Followed-up } & 53 & 40 & 35 & 34 \\ \text { Overlooked } & 45 & 31 & 26 & 23\end{array}$

Fig. 2: Association between the outcome of the initial visit in primary care and disease-specific death in a cohort of 221 head and neck carcinoma patients in Finland. At 3 years, $31 \%$ of patients who were referred for further care had died ( $95 \%$ confidence interval $[\mathrm{Cl}] 23 \%-39 \%), 30 \%$ of those who were followed up had died ( $95 \% \mathrm{Cl} 17 \%-43 \%$ ), and $45 \%$ of those whose disease was overlooked had died ( $95 \% \mathrm{Cl} 30 \%-60 \%)$. The absolute increase in the risk of death was $14 \%$ ( $95 \% \mathrm{Cl}-3$ to $31, p$ $=0.027$ ) between referred patients and those overlooked, and $-1 \%(95 \% \mathrm{Cl}-16$ to $14, p=0.97)$ between the patients referred and those followed up, by log-rank test). suspicion of cancer was associated with a delayed second consultation in primary care among patients whose head and neck cancer was initially missed. ${ }^{27}$

We had a population-based sample, but we did not find data on 46 cases, which makes a selection bias possible. Since ours was a retrospective sample, we had no control over the nature and quality of the primary care records. Nevertheless, these records were detailed and enabled us to obtain accurate information about the initial visit. Furthermore, these records were made before the patients knew they had cancer. The fact that we did not have a randomized trial leaves a question of how well groups with different cancers can be compared. The effect of overlooking the disease at initial presentation remained significant after adjustment for the other major prognostic variables, but unmeasured confounding may have had an influence. The effect may also be attributable to a lead time effect ${ }^{22}$ or to inaccuracies in determining whether death was caused by the disease. Here, however, the effect remained significant after both calculation of survival from symptom onset and use of all-cause death as the end point. The treatment of head and neck cancer in the study area and Finnish health care in general remained unchanged over the study period, but still, to adjust for any confounding effect of calendar time, the study year was included in the Cox multivariate model. In view of the characteristics of these carcinoma cases and the incidence and survival of head and neck cancer cases in Finland in general, ${ }^{28,29}$ our results are generalizable to other developed countries.

Despite recent advances in therapy, head and neck cancer continues to have a high death rate and often devastating treatment consequences for survivors. ${ }^{30,31}$ Because screening programs are not likely to increase survival, ${ }^{32}$ early detection in response to a symptom remains the choice for secondary prevention. There, the possible benefits of prompt referral of patients with cancer must be balanced with the risks and costs of unnecessary referral of those with benign tumours. According to our results, patients with tongue and glottic cancer would benefit from rapid referral. The diagnosis of head and neck cancer continues to be made by history-taking

Table 3: Association between outcome of initial primary care visit and disease-specific death at 3 years after diagnosis in a cohort of 221 patients with head and neck carcinoma

\begin{tabular}{|c|c|c|c|c|c|}
\hline \multirow[b]{2}{*}{$\begin{array}{l}\text { Patient group } \\
\text { by type of cancer }\end{array}$} & \multirow[b]{2}{*}{$\begin{array}{c}\text { Stage I or II at } \\
\text { diagnosis, }{ }^{20} \text { no. (\%) }\end{array}$} & \multicolumn{2}{|c|}{ Patient deaths by outcome, no. (\%) } & \multirow[b]{2}{*}{$\begin{array}{c}\text { Crude difference, } \\
\quad \%(95 \% \mathrm{Cl})\end{array}$} & \multirow[b]{2}{*}{ Adjusted HR† $(95 \% \mathrm{Cl})$} \\
\hline & & $\begin{array}{l}\text { Referred or } \\
\text { followed up* } \neq\end{array}$ & Overlooked ${ }^{*} \ddagger$ & & \\
\hline Tongue or glottic carcinoma & 72 (59) & $\begin{array}{l}n=98 \\
12(13)\end{array}$ & $\begin{array}{l}n=24 \\
11(46)\end{array}$ & $33(15$ to 52$)$ & $4.25(1.59-11.4)$ \\
\hline $\begin{array}{l}\text { Pharyngeal or } \\
\text { sub/supraglottic carcinoma }\end{array}$ & $23(23)$ & $\begin{array}{l}n=78 \\
39(54)\end{array}$ & $\begin{array}{l}n=21 \\
9(44)\end{array}$ & $-10(-34$ to 15$)$ & $1.15(0.49-2.68)$ \\
\hline All patients & $95(43)$ & $\begin{array}{l}n=176 \\
51(31)\end{array}$ & $\begin{array}{l}n=45 \\
20(45)\end{array}$ & 14 (0 to 29$)$ & $1.89(1.03-3.45)$ \\
\hline
\end{tabular}

Note: $\mathrm{Cl}$ = confidence interval, $\mathrm{HR}=$ hazard ratio.

*Referred = referred to hospital at the initial visit in primary care; followed up = scheduled follow-up visit arranged at the initial visit in primary care;

overlooked $=$ neither referred nor followed up at the initial visit in primary care.

†Adjusted for age, sex, socioeconomic status, duration of symptoms, cancer stage and study period.

$\ddagger$ Percentages calculated with the Kaplan-Meier method. 
and physical examination. Because of their rarity, malignant tumours may not be the first potential diagnosis in the physician's mind. Again, this appears often to result in insufficient physical examination and the lesions seen not being recognized as cancer. Our finding that initial misdiagnosis was not harmful if the patient was followed up indicates that a watchand-wait strategy may be used to rule out the possibility of cancer. In other words, physicians would do well to keep the possibility of cancer in mind, and, if not to make an immediate referral, at least to follow up with patients whose lesions are difficult to define conclusively. This would be particularly important with patients who present with persistent hoarseness, persistent ulcers or pain in the tongue.

In conclusion, the results of our study show that head and neck carcinoma is a rare disease with symptoms that are common in primary care in Finland. Results of studies of the effect of early detection on outcomes are contradictory. Our results support a major role for initial diagnosis in primary care in tongue and glottic carcinomas but not in pharyngeal and supraglottic carcinomas. Tongue and glottic carcinomas tended to cause symptoms early, whereas pharyngeal and supraglottic tumours usually presented at an advanced stage. It seemed that it was the initial decision not to refer or follow-up that was associated with poor survival, whereas initial misdiagnosis was not harmful if scheduled follow-up was arranged.

This article has been peer reviewed.

From the departments of Otorhinolaryngology (Alho, Teppo) and Diagnostics and Oral Medicine (Kantola), University of Oulu, and the Department of Public Health and General Practice (Mäntyselkä), General Practice Unit, University Hospital of Kuopio, Finland

\section{Competing interests: None declared.}

Contributors: Olli-Pekka Alho contributed to the conception and design of the study, the acquisition, analysis and interpretation of the data, and the drafting and revision of the article. Heikki Teppo, Pekka Mäntyselkä and Saara Kantola contributed to the acquisition, analysis and interpretation of the data and to critical revision of the article. All of the authors approved the final version.

Acknowledgements: We are indebted to Dr. Niko Rantala for collecting part of the data from the primary care files.

\section{REFERENCES}

I. Vokes EE, Weichselbaum RR, Lippman SM, et al. Head and neck cancer. N Engl J Med I993;328:184-94.

2. Franceschi S, Levi F, Vecchia C. Decline in 5-year survival rates for cancer of head and neck. Lancet I992;340:47.

3. Muir C, Weiland L. Upper aerodigestive tract cancers. Cancer 1995;75(Suppl I):I47-53.

4. Brenner H. Long-term survival rates of cancer patients achieved by the end of the 2oth century: a period analysis. Lancet 2002;360:1131-5.

5. Million RR, Cassisi NI, Clark JR. Cancer of the head and neck. In: DeVita VT, Hellman S, Rosenberg SA, eds. Cancer: principles and practice in oncology. Philadelphia: JB Lippincott; I989. p. 488-59o.

6. Dolan RW, Vaughan CW, Fuleihan N. Symptoms in early head and neck cancer: an inadequate indicator. Otolaryngol Head Neck Surg I998;119:463-7.

7. Guggenheimer J, Verbin RS, Johnson JT, et al. Factors delaying the diagnosis of oral and oropharyngeal carcinomas. Cancer I989;64:932-5.

8. Allison P, Franco E, Black M, et al. The role of professional diagnostic delays in the prognosis of upper aerodigestive tract carcinoma. Oral Oncol I998;34:147-53.

9. Allison P, Franco E, Feine J. Predictors of professional diagnostic delays for upper aerodigestive tract carcinoma. Oral Oncol ig98;34:127-32.

Io. Klein HC. Why can't physicians examine the larynx? JAMA I982;247:2III.

II. Ho T, Zahurak M, Koch WM. Prognostic significance of presentation-to-diagnosis interval in patients with oropharyngeal carcinoma. Arch Otolaryngol Head Neck Surg 2004;130:45-51.
I2. Koivunen P, Rantala N, Hyrynkangas K, et al. The impact of patient and professional diagnostic delays on survival in pharyngeal cancer. Cancer 200I;92:2885-9I.

13. Tromp DM, Brouha XD, Hordijk GJ, et al. Patient and tumour factors associated with advanced carcinomas of the head and neck. Oral Oncol 2005;41:313-9.

I4. Teppo H, Koivunen P, Hyrynkangas K, et al. Diagnostic delays in laryngeal carcinoma: professional diagnostic delay is a strong independent predictor of survival. Head Neck 2003;25:389-94.

I5. Mäntyselkä P. Patient pain in general practice. Medical Sciences I65. Kuopio: Kuopio University Publications; 1998.

I6. The World Organisation of National Colleges; Academies and Academic Associations of General Practitioners/Family Physicians (WONCA). (ICPC) International classification of primary care. Oxford: Oxford University Press; I99o.

17. Teppo L, Pukkala E, Lehtonen M. Data quality and quality control of a populationbased cancer registry. Experience in Finland. Acta Oncol 1994;33:365-9.

I8. International Union Against Cancer. TNM classification of malignant tumours. $4^{\text {th }}$ ed. Berlin: Springer-Verlag; 1987.

19. Recommendations for the Ig80 censuses of population and housing: the ECE region. Statistical standards and studies. New York: United Nations; 1978.

20. Kaplan EL, Meier P. Nonparametric estimation from incomplete observations. Am Stat Assoc 1958;53:457-8I.

2I. Evans SJ, Langdon JD, Rapidis AD, et al. Prognostic significance of STNMP and velocity of tumor growth in oral cancer. Cancer I982;49:773-6.

22. Gluckman JL, Zitsch RP. Early detection and screening for head and neck cancer. In: Hong WK, Weber RS, eds. Head and neck cancer. Basic and clinical aspects. Boston: Kluwer Academic Publishers; I995. p. I4I-57.

23. Mantel N. Evaluation of survival data and two new rank order statistics arising in its consideration. Cancer Chemother Rep i966;50:163-70.

24. Altman DG, Andersen PK. Calculating the number needed to treat for trials where the outcome is time to an event. BMJ I999;3I9:I492-5.

25. Cox DR. Regression models and life-tables (with discussion). J R Stat Soc [Ser A] I972;34:187-220.

26. Forastiere A, Koch W, Trotti A, et al. Head and neck cancer. N Engl J Med 20or; 345:1890-900.

27. Tromp DM, Brouha XD, Hordijk GJ, et al. Patient factors associated with delay in primary care among patients with head and neck carcinoma: a case-series analysis. Fam Pract 2005;22:554-9.

28. International Agency for Research on Cancer World Health Organization; International Association of Cancer Registries. Cancer incidence in five continents. Lyon: IARC Scientific Publications; I997.

29. Dickman PW, Hakulinen T, Luostarinen T, et al. Survival of cancer patients in Finland I955-I994 [see comments]. Acta Oncol I999;38(Suppl I2):I-I03.

30. Forastiere AA, Goepfert H, Maor M, et al. Concurrent chemotherapy and radiotherapy for organ preservation in advanced laryngeal cancer. N Engl J Med 2003; 349:209I-8.

31. Bernier J, Domenge C, Ozsahin M, et al. Postoperative irradiation with or without concomitant chemotherapy for locally advanced head and neck cancer. $N$ Engl Med 2004;350:1945-52.

32. Franceschi S, Barzan L, Talamini R. Screening for cancer of the head and neck: If not now, when? Oral Oncol 1997;33:313-6.

Correspondence to: Dr. Olli-Pekka Alho, Department of

Otorhinolaryngology, P.O. Box 5000, Oulu University, FIN-9ooI4, Finland; opalho@cc.oulu.fi

\section{Editor's take}

- Head and neck cancers have high death rates because the disease is often at an advanced stage before it is diagnosed. Does detection in primary care affect the outcome for patients with the disease?

- In this study, patients with tongue and glottic cancer whose initial presenting symptoms were overlooked by their primary care physician were at increased risk of death at 3 years compared with patients who were initially referred or followed up.

Implications for practice: Referral to otorhinolaryngologist or close follow-up should be considered for patients presenting to their primary care physician with symptoms of tongue and glottic cancer. 